\title{
МОДЕЛИРОВАНИЕ ВЛИЯНИЯ КОЭФФИЦИЕНТА НАПРАВЛЕННОГО ДЕЙСТВИЯ НА ТОЧНОСТЬ ПЕЛЕНГАЦИИ МЕТОДОМ МUSIC КОЛЬЦЕВОЙ АНТЕННОЙ РЕШЕТКИ
}

\author{
Ю. Б. Нечаев ${ }^{\star}$ И. В. Пешков ${ }^{\star *}$, К. Д. Аль Хафаджи Сармад \\ ${ }^{*}$ Воронежский государственный университет \\ ${ }^{*}$ Елецкий государственный университет
}

\section{Поступила в редакцию 19.02.2018 г.}

\begin{abstract}
Аннотация. Исследовано влияние коэффициента направленного действия отдельного антенного элемента на точность определения угловых координат источников радиоизлучения кольцевой антенной решеткой, состоящей из направленных излучающих элементов. Определены пределы изменения КНД отдельного элемента, при которых достигается максимальная точность оценки угловых координат. Показано, что при дальнейшем увеличении КНД возрастает СКО.

Ключевые слова: компьютерное моделирование, сверхразрешение, цифровая антенная решетка, радиопеленгация, кольцевые антенные решетки, направленные излучатели, кнд. Annotation. The article discusses the functioning of the radio direction-finding methods in the system of circular antenna arrays formed of the designed radiators, directional factor which is not equal to 1 . There estimate the accuracy of determining the angular coordinates with the direction-finding method with superresolution MUSIC.

Keywords: super resolution; antenna array; DOA estimation; circular arrays; directive antennas, directional factor.
\end{abstract}

\section{ВВЕДЕНИЕ}

В задачах радиолокации оценка угловых координат источников радиоизлучения (ИРИ) является ключевой для разделения целей, в то время как в беспроводных телекоммуникационных системах информация о координатах позволяет осуществить пространственное разделение для увеличения одновременно функционирующих пользователей [1].

В статье основное внимание уделяется определению влияния характеристик антенных элементов на получение достижимой точности при оценке угловых координат источников радиоизлучения. В то время как эквидистантные линейные антенные решетки преимущественно рассматриваются в литературе при описании формирования луча и радиопеленгации, равномерные кольцевые

() Нечаев Ю. Б., Пешков И. В., Аль Хафаджи Сармад К. Д., 2018 антенные решетки являются привлекательной альтернативой, когда необходимо получить более равномерные характеристики по всем углам обзора. Подавляющее большинство публикаций по адаптивным антеннам, затрагивающих темы оценки угловых координат и диаграммообразования, предполагают, что используются близкие к изотропным антенные элементы, где усиление антенны постоянно во всех направлениях (например, полуволновые диполи) [2-7]. Поэтому рассмотрение влияния такой характеристики антенных элементов, как коэффициент направленного действия (КНД), на характеристики радиопеленгации представляют серьезный интерес.

\section{ОСНОВНЫЕ ПРЕДПОЛОЖЕНИЯ}

Предположим, что $M$ радиосигналов приходят на антенную решетку с различных направлений $\left\{\varphi_{m}, \theta_{m}\right\}_{m=0}^{M-1}$, где $\varphi$ - угол места, $\theta-$ азимут. Для произвольной геометрической 
Марковские модели немарковских процессов для множественных рекуррентных потоков ...

конфигурации комплексный вектор сигналов на выходе АР описывается выражением:

$$
\overrightarrow{\mathbf{x}}(t)=\mathbf{A} \cdot \overrightarrow{\mathbf{s}}(t)+\overrightarrow{\mathbf{n}}(t),
$$

где $\overrightarrow{\mathbf{x}}(t)-N$-мерный вектор, описывающий сигналы на выходе каждого антенного элемента $\mathrm{AP}, \overrightarrow{\mathbf{s}}(t)-M$-мерный вектор сигналов; $\overrightarrow{\mathbf{n}}(t)-N$-мерный вектор шума, $\mathbf{A}-N \times M$ матрица направляющих векторов, $m$-й столбец которой соответствует фазовому и амплитудному распределению на АР $m$-го сигнала. В общем виде один из векторов матрицы А выглядит как [8]:

$$
\mathbf{a}(\theta)=\left[\begin{array}{c}
g_{0}(\theta) e^{j \beta r \cos \theta} \\
g_{1}(\theta) e^{j \beta r \cos \left(\theta-\frac{2 \pi}{N}\right)} \\
g_{2}(\theta) e^{j \beta r \cos \left(\theta-\frac{4 \pi}{N}\right)} \\
\cdots \\
g_{N-1}(\theta) e^{j \beta r \cos \left(\theta-\frac{2(N-1) \pi}{N}\right)}
\end{array}\right],
$$

где $\beta=\frac{2 \pi}{\lambda}-$ волновое число или коэффициент распространения волны ( $\lambda$ - длина волны), $g_{0}(\theta), g_{1}(\theta), \ldots, g_{N-1}(\theta)$ - действительные числа, представляющие амплитуду отклика антенного элемента (т. е. усиление антенны) в направлении $\theta$.

Пусть антенные элементы в составе кольцевой АР идентичны и имеют максимум в направлении радиально от центра решетки, $g\left(\varphi-\frac{2 \pi n}{N}\right), n=0,1, \ldots, N-1$. В этом случае направляющий вектор задается как [8]:

$$
\mathbf{a}(\theta)=\left[\begin{array}{c}
g(\theta) e^{j \beta r \cos \theta} \\
g\left(\theta-\frac{2 \pi}{N}\right) e^{j \beta r \cos \left(\theta-\frac{2 \pi}{N}\right)} \\
g\left(\theta-\frac{4 \pi}{N}\right) e^{j \beta r \cos \left(\theta-\frac{4 \pi}{N}\right)} \\
\cdots \\
g\left(\theta-\frac{2(N-1) \pi}{N}\right) e^{j \beta r \cos \left(\theta-\frac{2(N-1) \pi}{N}\right)}
\end{array}\right]
$$

где $\beta=\frac{2 \pi}{\lambda}-$ волновое число или коэффициент распространения волны ( $\lambda$ - длина волны), $g(\cdot)$ - неотрицательная гладкая периодическая функция с периодом $2 \pi$.
Следует отметить, что поскольку направляющий вектор (1) представляет амплитудный и фазовый отклики антенн в составе решетки от различных падающих волн, то усиление $g$ является коэффициентом усиления по напряжению (или тока) относительно значений, которые могли бы быть приняты гипотетической изотропной антенной. Антенны обычно определяются в терминах их излучаемой (или принимаемой) мощности в определенном направлении по отношению к изотропной антенне. Если диаграмму направленности (ДН) антенны по мощности обозначить как линейное усиление $G(\theta)$ относительно изотропной антенны, тогда $g(\theta)=\sqrt{G(\theta)}$.

Предположим, что $\overrightarrow{\mathbf{s}}(t)$ и $\overrightarrow{\mathbf{n}}(t)$ - стационарные случайные процессы, $\overrightarrow{\mathbf{n}}(t)$ - гауссовский случайный процесс с нулевым средним и ковариационной матрицей $\sigma^{2} \mathbf{I}\left(\sigma^{2}-\right.$ дисперсия шума), а также, что сигналы некоррелированы и отсутствует корреляция между шумом и сигналами. Тогда пространственная корреляционная матрица (КМ) в общем виде может быть записана:

$$
\mathbf{R}=E\left[\overrightarrow{\mathbf{x}}(t) \overrightarrow{\mathbf{x}}^{H}(t)\right]=\mathbf{A S A}^{H}+\sigma^{2} \mathbf{I},
$$

где $E[\ldots]$ - математическое ожидание, $(\ldots)^{H}-$ эрмитово сопряжение, $\mathbf{S}=E\left[\overrightarrow{\mathbf{s}}(t) \overrightarrow{\mathbf{s}}^{H}(t)\right]-$ корреляционная матрица сигналов.

Сигнал создается несколькими взаимно некоррелированными внешними источниками и собственным шумом:

$$
\overrightarrow{\mathbf{x}}(t)=\sum_{m=0}^{M-1} s_{m}(t) \overrightarrow{\mathbf{a}}\left(\theta_{m}\right)+\overrightarrow{\mathbf{n}}(t) .
$$

Некоррелированность сигналов отдельных источников приводит к тому, что КМ будет равна сумме КМ отдельных источников и шума:

$$
\begin{gathered}
\quad \mathbf{R}=\sum_{m=0}^{M-1} E\left[s_{m}^{2}(t)\right] \overrightarrow{\mathbf{a}}^{H}\left(\theta_{m}\right) \overrightarrow{\mathbf{a}}\left(\theta_{m}\right)+\sigma_{0}^{2} \mathbf{I}= \\
=\sum_{m=0}^{M-1} p_{m} \overrightarrow{\mathbf{a}}^{H}\left(\theta_{m}\right) \overrightarrow{\mathbf{a}}\left(\theta_{m}\right)+\sigma^{2} \mathbf{I}=\mathbf{A S} \mathbf{A}^{H}+\sigma^{2} \mathbf{I} .
\end{gathered}
$$

Для некоррелированных источников она представляет собой диагональную матрицу [6]:

$$
\mathbf{S}_{i j}=\left\{\begin{array}{l}
p_{i}, i=j \\
0, i \neq j
\end{array},\right.
$$

где $p_{i}$ - мощность $i$-го сигнала. 
Поскольку ранг матрицы $\mathbf{A S A}^{H}$ равен $M$, тогда $\mathbf{A S A}^{H}$ имеет $M$ положительных собственных значений, а остальные $(N-M)$ собственные значения равны нулю. Из этого следует, что для матрицы $\mathbf{A S A}^{H}+\sigma_{0}^{2} \mathbf{I}$ собственные значения: $\lambda_{1}>\lambda_{2}>\ldots>\lambda_{M}>\sigma^{2}$ и $\lambda_{M+1}=\ldots=\lambda_{N}=\sigma^{2}$. Тогда множество собственных значений можно разделить на два подмножества, каждое из которых обладает уникальными свойствами.

Пусть $\lambda_{1} \geq \lambda_{2} \geq \ldots \geq \lambda_{N-1} \geq \lambda_{N}-$ собственные значения матрицы $\mathbf{R}$. Пусть также $\mathbf{E}_{s}=\left[\overrightarrow{\mathrm{e}}_{1}, \overrightarrow{\mathrm{e}}_{2}, \ldots, \overrightarrow{\mathrm{e}}_{M}\right]-$ ортонормированные собственные векторы, соответствующие $M$ самым большим собственным значениям $\Lambda_{\mathrm{s}}=\left[\lambda_{1}, \lambda_{2}, \ldots, \lambda_{M}\right] \quad$ и $\quad \mathbf{E}_{n}=\left[\overrightarrow{\mathrm{e}}_{M+1}, \overrightarrow{\mathrm{e}}_{M+2}, \ldots, \overrightarrow{\mathrm{e}}_{N}\right]-$ матрица, состоящая из ортонормированных собственных векторов, соответствующих $(N-M)$ самым малым собственным значениям $\Lambda_{n}=\left[\lambda_{M+1}, \lambda_{M+2}, \ldots, \lambda_{N}\right]$. Пусть

$$
\mathbf{R E}_{n}=\Lambda_{n} \mathbf{E}_{n}=\sigma_{0}^{2} \mathbf{E}_{n}=\mathbf{A S A}^{H} \mathbf{E}_{n}+\sigma^{2} \mathbf{E}_{n},
$$
тогда следует, что $\mathbf{A}^{H} \mathbf{E}_{n}=0$. Другими словами, столбцы матрицы $\mathbf{E}_{n}$ принадлежат нуль-пространству $\mathbf{A}^{H}$. Поскольку собственные векторы шумового подпространства ортогональны к столбцам матрице направляющих векторов $\mathbf{A}$, что соответствует истинным координатам источников радиоизлучения $\left\{\varphi_{m}, \theta_{m}\right\}_{m=0}^{M-1}$, тогда пространственный спектр метода MUSIC [9]

$$
P_{\text {MUSIC }}(\varphi, \theta)=\frac{1}{\overrightarrow{\mathbf{a}}^{H}(\theta) \mathbf{E}_{N} \mathbf{E}_{N}^{H} \overrightarrow{\mathbf{a}}(\theta)} .
$$

Числитель (2) становится равным нулю, и выражение $P_{\text {MUSIC }}(\varphi, \theta)$ стремится к бесконечности соответствуя оценкам координат $\left\{\hat{\varphi}_{m}, \hat{\theta}_{m}\right\}_{m=0}^{M-1}$.

Для исследования эффекта влияния коэффициента направленного действия на точность радиопеленгации необходимо иметь модель диаграммы излучения гипотетической антенны. В данной статье проблемы и ограничения процесса разработки антенн не рассматриваются, цель работы - исследовать эффект влияния направленности АЭ на точность радиопеленгации. Модель диаграммы направленности антенного элемента в дальней зоне имеет отклик $(1+\cos (\theta))^{m}$ в азиму- тальной плоскости, где $m$ контролирует направленность. Такая ДН имеет КНД, который увеличивается с увеличением степени $m$ и имеет максимальное усиление в $\theta=0^{\circ}$. Данная модель является близкой аппроксимацией антенн, которые имеют излучение в прямом и обратном направлении, такие как микрополосковые антенны с конечным основанием. Кроме того, предполагается, что ДН симметрична в трехмерной плоскости, тогда нормализованная диаграмма для излучателей, расположенных по кольцу, представляется как [8]:

$$
\begin{gathered}
U_{n}(\phi, \theta)= \\
=\frac{1}{2^{2 m}}(1+\sin (\phi))^{m}\left(1+\cos \left(\theta-\frac{2 \pi n}{N}\right)\right)^{m}, \\
n=0,1, \ldots, N-1 .
\end{gathered}
$$

Необходимо отметить, что максимум ДН для каждого элемента направлен радиально от центра равномерно распределенной кольцевой АР. Используя математическую модель ДН (3), коэффициент направленного действия каждого антенной элемента в составе решетки вычисляется как [10]:

$$
D=\frac{2^{2 m+2} \pi}{\int_{0}^{2 \pi} \int_{0}^{\pi}(1+\sin (\phi))^{m}(1+\cos (\theta))^{m} \sin (\phi) d \phi d \theta}
$$

Для изотропной антенны, $m=0$ и $D=1$ в выражении (4) и при увеличении $m$ направленность $D$ также увеличивается. К примеру, когда $m \approx 2,7, D=4$ и когда $m \approx 8,7$, направленность $D=10$.

Используя выражение (4) можно получить математическую модель ДН по мощности в дальней зоне в плоскости при $\varphi=90^{\circ}$ относительно изотропной антенны в предположении, что антенны идеально согласованы и без потерь [10]:

$$
\begin{gathered}
G(\theta)=\frac{D}{2^{2 m}}\left(1+\cos \left(\theta-\frac{2 \pi n}{N}\right)\right)^{m}, \\
n=0,1, \ldots, N-1 .
\end{gathered}
$$

На рис. 2 показаны расчетные ДН гипотетической антенны для $\varphi=90^{\circ}$ с коэффициентами направленного действия $D=2,4,10,25$, 50 (т. е. от 3 дБи до 17 дБи), а также ДН изотропной АЭ для сравнения. 


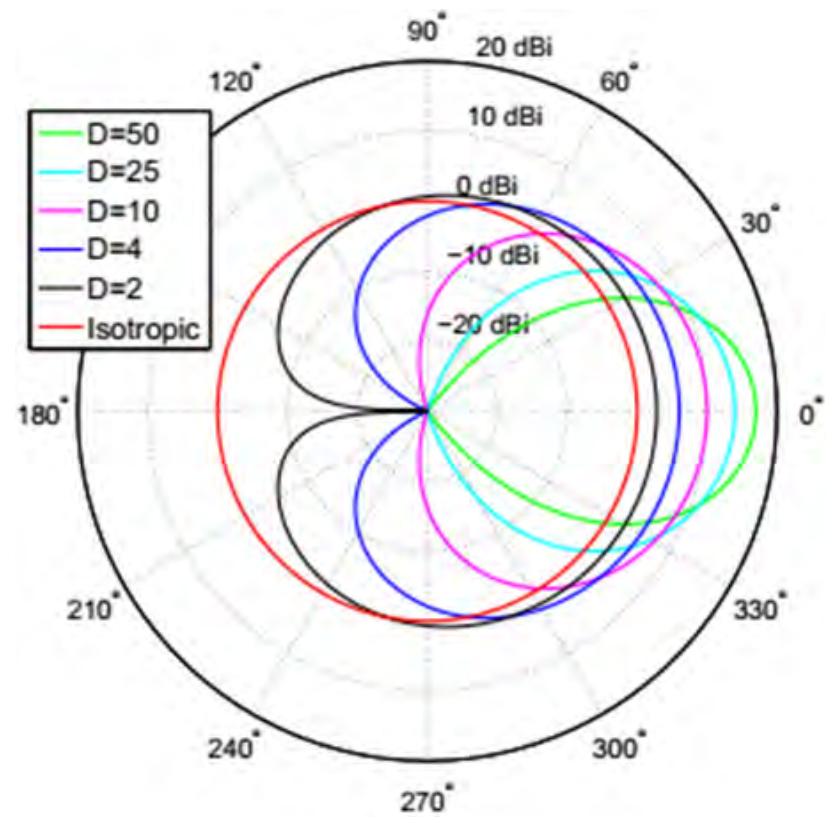

Рис. 1. Графики диаграмм направленности для различных $D=1,2,4,10,25,50, \varphi=90^{\circ}$

\section{ИССЛЕДОВАНИЕ}

Проведено исследование точности метода радиопеленгации со сверхразрешением MUSIC (2) в составе кольцевых антенных решеток в зависимости от коэффициента направленного действия антенных элементов. Такого рода антенные решетки, составленные из направленных излучателей, называются конформными. Диапазон изменения КНД протирался от 1 (всенаправленный излучатель) до 30. При числе излучателей, равном восьми, и при учете того, что ширина подложки составляет $\lambda / 2$, возьмем радиус $r=(1+\sqrt{2}) \lambda / 4$, тогда

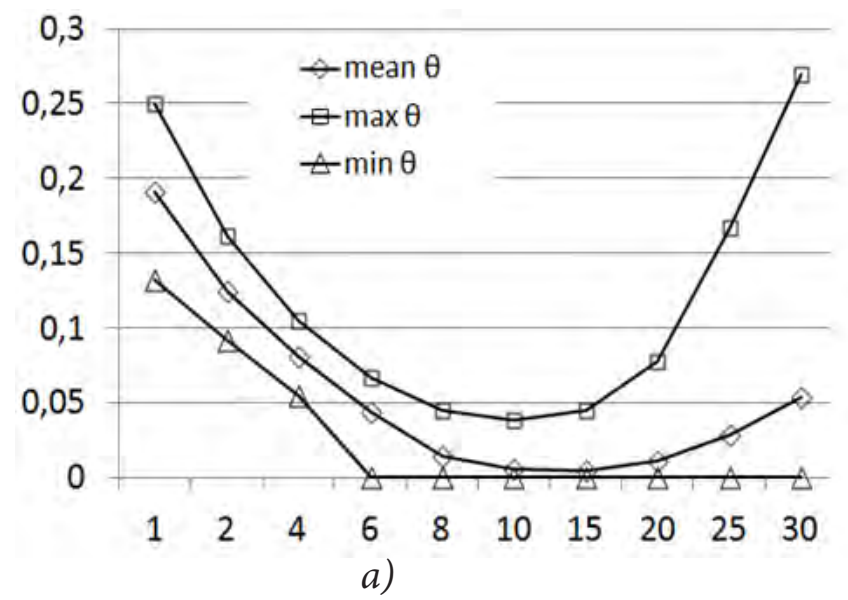

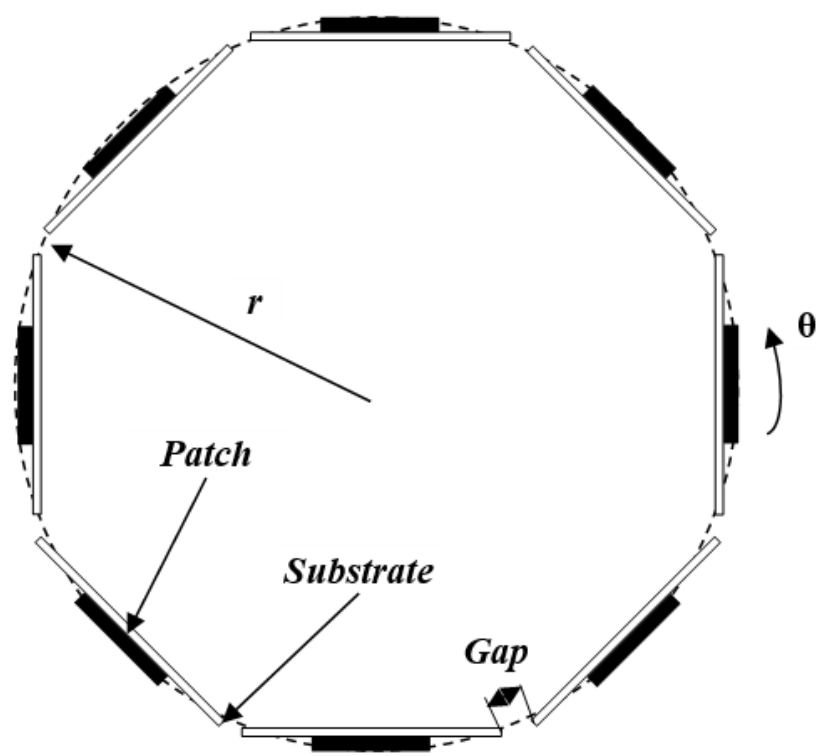

Рис. 2. Схема антенной решетки из восьми направленных излучателей, состоящих из подложки ("Substrate") и излучателя ("Patch") зазор (“Gap”) между элементами отсутствует (рис. 2), также рассмотрим конфигурацию с радиусом решетки $\lambda$.

Рассмотрим ситуацию с одним источником. Азимут источника сигнала смещался от $0^{\circ}$ до $180^{\circ}$. Вычислялось среднее значение среднеквадратического отклонения (СКО) по всем направлениям (“mean $\theta$ ”), а также максимальное ("max $\theta$ ") и минимальное значение ошибки (“min $\theta$ ").

Установлено, что при увеличении КНД от 1 до 10 среднее значение СКО уменьшалось от $0,2^{\circ}$ до $0,01^{\circ}$, при этом разброс между минимальным и максимальным СКО не превышал $\sim 0,05^{\circ}$, что говорит о равномерности по всем

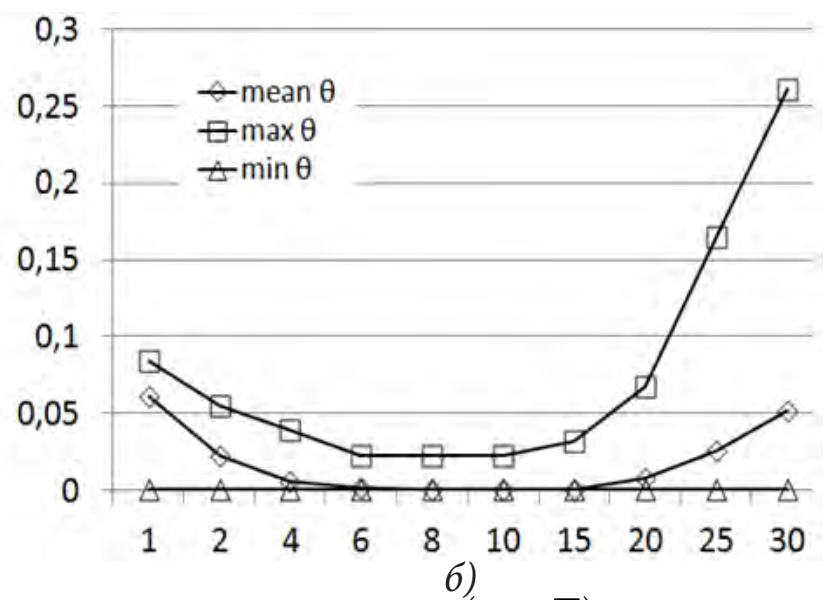

Рис. 3. Зависимость СКО от КНД для решеток с радиусами а) $r=(1+\sqrt{2}) \lambda / 4$ и б) $r=\lambda$ для одного сигнала 
Ю. Б. Нечаев, И. В. Пешков, К. Д. Аль Хафаджи Сармад
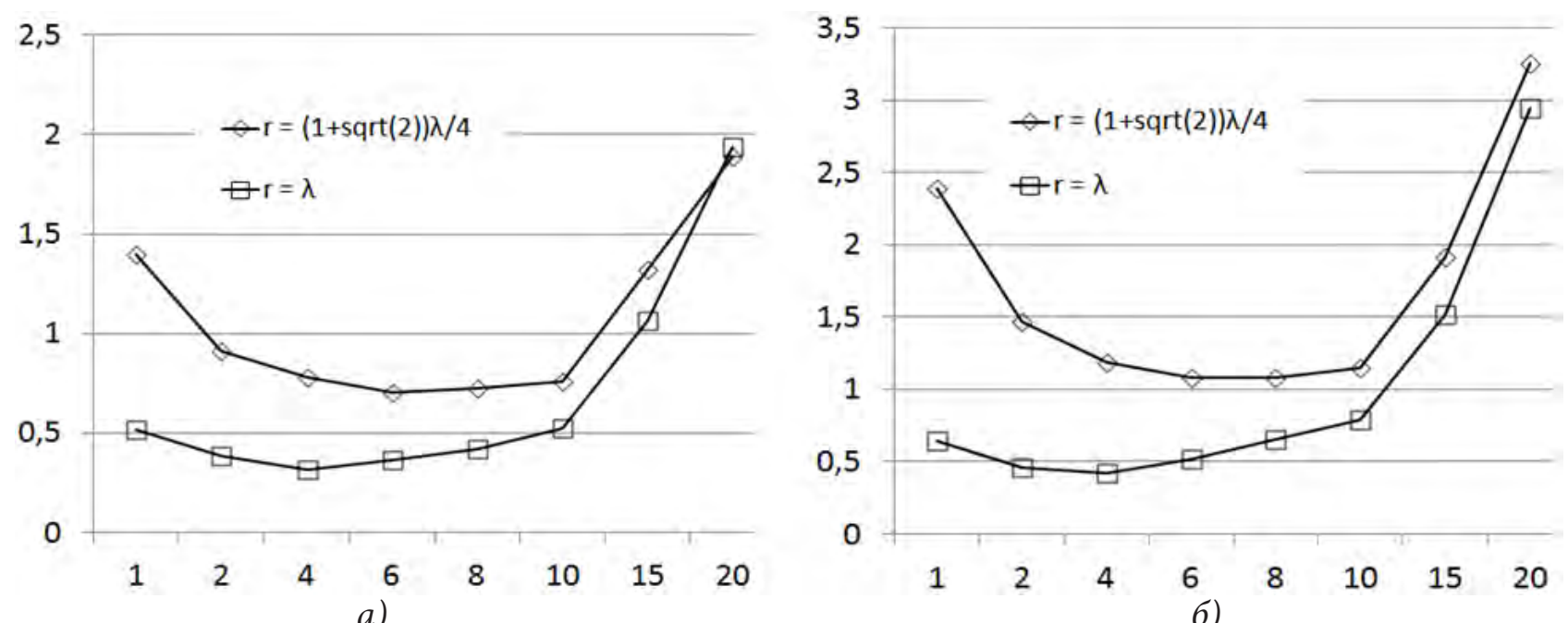

Рис. 4. Зависимость СКО от КНД для решеток с радиусами $r=(1+\sqrt{2}) \lambda / 4$ $u r=\lambda$ при а) двух и б) трех сигналах

направлениям оценок координат источника сигнала. При увеличении КНД от 10 до 30 среднее значение СКО увеличивалось до 0,05, при этом разброс между минимальным и максимальным СКО достигал $\sim 0,2^{\circ}$, что говорит о зависимости оценок координат источника сигнала от его направления относительно антенной решетки.

Рассмотрим ситуацию с несколькими источниками радиоизлучения. При этом вычислялось СКО для нескольких сигналов, координаты которых составляли $10^{\circ}$ и $25^{\circ}$, а также $10^{\circ}, 30^{\circ}$ и $60^{\circ}$ (рис. 4 ).

Из графиков на рис. 4 установлено, что при увеличении КНД от 1 до 10 СКО уменьшалось от $1,5^{\circ}$ до $0,75^{\circ}$ и от $2^{\circ}$ до $1^{\circ}$ соответственно при приходе волн от двух и трех источников на антенные решетки. При увеличении КНД от 10 до $30 \mathrm{CKO} \mathrm{увеличивалось} \mathrm{до} 2^{\circ}$ и $3^{\circ}$ соответственно.

\section{ЗАКЛЮЧЕНИЕ}

В статье исследовано влияние коэффициента направленного действия антенного элемента в составе кольцевой антенной решетки на точность процедуры оценки угловых координат методом MUSIC. Точность радиопеленгации со сверхразрешением сравнивается для идеальных изотропных элементов и для направленных элементов с полученной теоретически диаграммой направленности по мощно- сти, и с помощью компьютерной симуляции установлено, что значительное повышение точности может быть достигнуто, если использовать подходящее значение коэффициента направленного действия. Для оценки влияния КНД, изменяемого в пределах от 1 до 30, на точность радиопеленгации были использованы восьмиэлементные антенные решетки с радиусами $(1+\sqrt{2}) \lambda / 4$ и $\lambda$ при фиксированном отношении сигнал-шум 10дБ. Установлено, что оптимальным выбором коэффициента направленного действия для высокой точности является значение в пределах 6-8. При дальнейшем увеличении этой величины СКО метода MUSIC значительно повышается, а также возрастает зависимость ошибки пеленгации от азимутального положения источника сигнала, поскольку разброс между максимальным и минимальным СКО $0,25^{\circ}-0,3^{\circ}$.

\section{СПИСОК ЛИТЕРАТУРЫ}

1. Krim H. Two decades of array signal processing research / H. Krim, M. Viberg // IEEE Signal Processing Magazine. - 1996. - V. 7. P. 67-94.

2. Trees Van H. L. Detection, Estimation, and Modulation Theory, Optimum Array Processing / H. L. Trees Van. - John Wiley \& Sons, 2002. $-1470 \mathrm{p}$.

3. Liu J., Li L. and Huazhi W. Investigation of different types of array structures for smart an- 
Марковские модели немарковских процессов для множественных рекуррентных потоков ...

tennas // Int. Conf. on Microwave and Millimeter Wave Technology (ICMMT). - 2008. - P. 11601163.

4. Islam M. R. and Adam I. A. H. Perfomance study of direction of arrival (DOA) estimation algorithms for linear array antenna // Int. Conf. on Signal Processing Systems. - 2009. - P. 268-271.

5. $W u$ B. Realization and simulation of DOA estimation using MUSIC algorithm with uniform circular arrays // The 4th Asia-Pacific Conf. on Environmental Electromagnetics. - 2006. P. 908-912.

6. Rui L., Xiaowei S., Lei C., Ping L. and Le X. The non-circular MUSIC method for uniform rectangular arrays // Int. Conf. on Microwave and Millimeter Wave Technology (ICMMT). 2010. - P. 1390-1393.

Нечаев Ю. Б. - д-р физ.-мат. наук, профессор, профессор кафедры информационных систем, Воронежский государственный университет.

E-mail: nechaev_ub@mail.ru

Пешков И. В. - доцент кафедры радиоэлектроники и компьютерной техники, Елецкий государственный университет.

E-mail: ilvpeshkov@gmail.com

Аль Хафаджи Сармад К. Д. - аспирант кафедры информационных систем, Воронежский государственный университет.

E-mail: Sarmad_kad@yah00.com
7. Nechaev Yuri, Peshkov Ilia. Accuracy Researching of Direction-of-Arrival Estimation via Music for Circular, Octagonal, Hexagonal And Rectangular Antenna Arrays // International Journal of Research in Engineering and Science. September 2016. - V. 4, Issue. 9. - P. 08-15.

8. Jackson B. R. Direction of Arrival Estimation Using Directive Antennas in Uniform Circular Arrays / Jackson Brad R., Rajan Sreeraman, Liao Bruce J., Wang Sichun // EEE Transactions on Antennas and Propagation. - V. 63, issue 2. P. 736-747.

9. Schmidt R. O. Multiple emitter location and signal parameter estimation // IEEE Transactions on Antennas and Propagation. - 1986. - 34 (3). 276-280.

10. Kraus J. D. Antennas. McGraw-Hill, 1988.

Nechaev Yu. B. - Dr. Sci. (Phys.-Math.), Prof., professor of information systems department of the Voronezh State University.

E-mail: nechaev_ub@mail.ru

Peshkov I. V. - Cand. Sci. (Phys.-Math.), senior teacher of Radio electronics and Computer Science department of the Elets State University. E-mail: ilvpeshkov@gmail.com

Alkhafaji Sarmad K. D. - graduate student of department of Information Systems Voronezh State University.

E-mail: Sarmad_kad@yah00.com 